\title{
Antifeedant Activity of Sesquiterpenes from Celastraceae
}

\author{
ANTONIO G. GONZALEZ, `§ IGNACIO A. JIMENEZ," ANGEL. G. RAVELO," JOSEP COLL, $†$ \\ JOSE A. GONZALEZ \\ -Centro de Productos Naturales Orgánicos "Antonio González", Instituto Universitario de Bio-Orgánica, \\ Universidad de La Laguna, Carretera La Esperanza 2, La Laguna, E-38206 Tenerife, Canary Islands; \\ tCentro de Investigación y Desarrollo, CSIC, Jorge Girona Salgado 18-26, E-08034 Barcelona, Spain: \\ tDpt. de Agroecología, Centro de Ciencias Medioambientales, CSIC, Serrano 115 dpdo, E-28006 Madrid,
}

Spain

Key Word Index-Celastraceae; antifeedant activity; insecticidal activity; structure-activity relationship; dihydro- $\beta$-agarofuran sesquiterpenes; Spodoptera littoralis.

Abstract-Forty one sesquiterpenes with a dihydro- $\beta$-agarofuran skeleton, and 14 related synthetic compounds are assayed for antifeedant and insecticidal activities against the Egyptian cotton leafworm Spodoptera fittoralis using the leaf disk method. The antifeedant activity is measured in terms of feeding ratio (FR $\left.{ }_{60}\right)$ and the insecticidal activity as lethal dose $L_{100}$. Thirty eight of the sesquiterpenes assayed show antifeedant activity, including $\mathbf{3 8}$ more active then triphenyl tin acetate used as a positive control in this assay. Three sesquiterpenes demonstrate insecticidal activity. Structure activity relations are examined.

\section{Introduction}

Clerodane diterpenes from various plant species (Kato et al., 1972; Bellés et al., 1985), drimane-type sesquiterpenes from Warburgia species (Kubo et al., 1976), and limonoids such as azadirachtin from the Meliaceae (Ley, 1990) are some of the best studied natural products with insect antifeedant activity. For the clerodane diterpenes and azadirachtins, bioactivity has been related to specific structural features of the molecule. The presence of a furan or perhydrofuran moiety may be in part responsible for their antifeedant activity (Kojima and Kato, 1981), although when the furan system is replaced by a butenolide ring in some clerodanes, feeding is in fact further inhibited (Kubo et al., 1976). Antifeedant activity has also been associated with the presence of a decalin ring system and with ester moieties (Geuskens et al., 1983) but there is no clear indication of how structure is related with activity and mode of action.

Various species of Celastraceae have been used in Chinese traditional agriculture to protect crops from insect attack (Swingle et al., 1941). The Chinese bittersweet Celastrus angulatus Max. has demonstrated activity against several insect species (Wakabayashi et al., 1988) and there is a body of work on the antifeedant or insecticidal activity of extracts (Chiu, 1989), or of isolated products such as angulatin A (Maotian et al., 1991), celangulins (Wakabayashi et al., 1988; Wu et al., 1992) and other sesquiterpenes (Kai et al., 1991).

Sesauiteroenes with a dihvdro- $\beta$-aaarofuran skeleton (5.11-eboxv-5B.10 $\alpha$-eudesm- 
(Brüning and Wagner, 1978). In a preliminary study we reported the antifeedant activity of 15 polyesterified sesquiterpenes from Celastraceae against the Egyptian cotton leafworm Spodoptera littoralis (González et al., 1992a).

This paper reports the activity and structure-activity relations of 41 sesquiterpenes isolated from seven species of Celastraceae and 14 related synthetic compounds on the fifth-instar larvae of $S$. littoralis.

\section{Materials and Methods}

Insects. Newly ecdysed fifth-instar larvae of the Egyptian cotton leafworm Spodoptera littoralis Boisduval, taken from a laboratory culture were used for the antifeedant bioassays. The larvae were reared on a semi-artificial diet (Poitout and Bies, 1974) at $25 \pm 2^{\circ} \mathrm{C}, 60-70 \%$ relative humidity and $18 \mathrm{~h}$ photophase.

Compounds tested. Compounds 1-41 (Fig. 1), were isolated by repeated column chromatography on silica gel or Sephadex LH-20 from the EtOH extracts of seven Celastraceae species. All compounds were rigorously characterized by spectroscopic methods (UV, IR, ${ }^{1} \mathrm{H}$ MNR, ${ }^{13} \mathrm{C}$ NMR, HMBC. HMQC, MS) and chemical data. Compounds 1 (González et al., 1992b), 11-13, 31-33 (González et al., 1994a) were isolated from Maytenus boaria; compounds 3, 38-40 (González et al., 1993b), 16, 17, 20 (González et al., 1990), 19, 35, 36 (González et al., 1989a), 21, 22, 34 (González et al., 1994b) , 18, 23, 25 (González et al., 1993a), 24 (Jiménez, 1992) and 37 (celangulin) (Wakabayashi et al., 1988; González et al., 1993b) from Maytenus canariensis; compounds 7, 8, 14, 15, 26, 27 (González et al., 1994a) from Maytenus chubutensis; compounds 9, 10 (González et al., 1994a) from Maytenus disticha; compounds 28-30 (González et al., 1994a) from Maytenus magellanica; compounds 4, 5 (González et al., 1988) and 41 from Orthosphenia mexicana (González et al., 1989b) and compounds 2 (González et al., 1987) and 6 (González et al., 1988) from Rzedowskia tolantonguensis.

Compounds 42-55 were racemic and/or optically active tetrahydrofuran synthetic compounds with different substituents on $\mathrm{C}-2$ or $\mathrm{C}-3$ and 2 -methylbutanoic acid and derivatives (Fig. 2).

Triphenyl tin acetate (TTA) (Aldrich; 97\%) was used as positive control, as it has antifeedant activity against S. littoralis (Bellés and Piulachs, 1983).

Antifeedant activity. The technique used was a choice test (Bellés and Piulachs, 1983; Bellés et al., 1985) using lettuce disks of $1 \mathrm{~cm}^{2}(33.65 \pm 0.07 \mathrm{mg} \mathrm{fwt})$. The test compounds were uniformly distributed over the surface of the disk by application of $10 \mu \mathrm{g}$ of compound in acetone $(10 \mu \mathrm{l})$ that was allowed to evaporate to give the treated disks (TD). Control disks (CD) were prepared in the same manner but without test compounds. For each bioassay, four TD and four CD were placed alternately in a Petri dish with five larvae (weight $40-50 \mathrm{mg}$ ) and kept in a growth chamber in the dark at $30^{\circ} \mathrm{C}, 70 \%$ relative humidity. There were six replicates for each compound tested. The consumed area of the treated disks (CTD) and those of the controls (CCD) was visually estimated on millimetre paper simultaneously at regular $30 \mathrm{~min}$ intervals for $4-5 \mathrm{~h}$ to calculate the feeding ratio $(F R=C T D / C C D)$. $F R_{50}$, the feeding ratio when the $C D$ had been $50 \%$ consumed, was used as a measure of antifeedant activity.

Insecticidal activity. Compounds that showed insecticidal activity in the antifeedant assay were further assayed. For each bio-assay, four TD with different doses of the compound $\left(10,1,0.1,0.01,0.005 \mu \mathrm{g} \mathrm{cm}^{-2}\right.$ ) and four $C D$ were placed in different Petri dishes with five larvae each. Experimental conditions were the same as those for the choice test and each test was repeated six times. Lethal dose of compounds for $100 \%$ mortality after $3 \mathrm{~h}$ exposure $\left(L D_{100}\right)$ was based on direct observation.

\section{Results and Discussion}

Among the 55 compounds tested, only the sesquiterpenes (1-41) were active, 38 (138) of which showed antifeedant activity and $3(39-41)$ insecticidal activity.

As shown in Table 1, sesquiterpenes 1-2, 4, 7, 11-14, 16, 18, 20, 27-29, 31-33, 35 and 38 exhibited antifeedant activity at $10 \mu \mathrm{g} \mathrm{cm}^{-2}$ in the choice test; $5,8-10,15,19$, $21,25-26$ and 30 at $1 \mu \mathrm{g} \mathrm{cm}^{-2} ; 3,6,22-24,34$ and $36-37$ at $0.1 \mu \mathrm{g} \mathrm{cm}{ }^{-2}$ and a remarkable activity was observed for compound 17 ( $9 \alpha$-benzoyloxy-8 $\alpha, 2$-methylbutyroyloxy-1 $\alpha, 6 \beta, 15$-triacetoxy- $4 \beta$-hydroxydihydro- $\beta$-agarofuran) with a $F R_{50}=0.69 \pm$ 0.08 at the lowest concentration tested of $0.01 \mu \mathrm{g} \mathrm{cm}^{-2}$.

Regarding structure-activity relations, we observed that the most active compounds were those with isoalatol $(17,22-24)$ or $4 \beta$-hydroxyalatol skeletons $(34,36-37)$ (Table 2). Comparing the activities of compounds with the same skeleton the activity increases with the number of acetate esters and decreases with the number of benzoate 


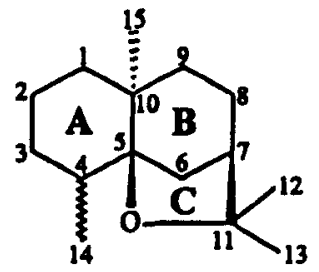

\begin{tabular}{|c|c|c|c|c|}
\hline Compound & $\mathrm{OH}$ & $\mathrm{OBz}$ & OAc & Others \\
\hline 1 & $3 \beta, 4 \alpha$ & & & \\
\hline 2 & $4 \beta$ & $1 \alpha$ & $9 \beta$ & $6 \beta$ (ONic) \\
\hline 3 & $4 \beta$ & $9 \beta$ & $1 \alpha, 6 \beta, 15$ & \\
\hline 4 & $4 \beta, 8 \beta$ & $1 \alpha$ & $9 \alpha$ & $6 \beta(O N i c)$ \\
\hline 5 & $4 \beta$ & $1 a$ & $6 \beta, 8 \beta, 9 \alpha$ & \\
\hline 6 & $4 \beta$ & $1 \alpha$ & $8 \beta, 9 \alpha$ & $6 \beta(\mathrm{ONic})$ \\
\hline 7 & & $9 \beta$ & $1 \alpha, 2 \alpha, 6 \beta, 15$ & \\
\hline 8 & 15 & $9 \beta$ & $1 \alpha, 2 \alpha, 6 \beta$ & \\
\hline 9 & & $9 \beta$ & $1 \alpha, 6 \beta, 8 \alpha, 15$ & \\
\hline 10 & $8 \alpha$ & $9 \beta$ & $1 \alpha, 6 \beta, 15$ & \\
\hline 11 & $3 \beta, 4 \beta$ & $1 \alpha, 9 \beta$ & $2 \beta$ & \\
\hline 12 & $3 \beta$ & $1 \alpha, 9 \beta$ & $2 \beta, 6 \beta$ & \\
\hline 13 & $4 \beta$ & $1 a, 9 \beta$ & $2 \beta, 6 \beta$ & \\
\hline 14 & 15 & $9 a$ & $1 \alpha, 2 \alpha, 6 \beta$ & $8(C=0)$ \\
\hline 15 & $4 \beta, 15$ & $9 a$ & $1 \alpha, 2 \alpha, 6 \beta, 8 \alpha$ & \\
\hline 16 & $4 \beta$ & $1 \alpha, 9 \alpha$ & $6 \beta, 15$ & $8 \alpha(\mathrm{OMeBut})$ \\
\hline 17 & $4 \beta$ & $9 a$ & $1 \alpha, 6 \beta, 15$ & $8 \alpha(O M e B u t)$ \\
\hline 18 & $4 \beta$ & $1 a, 8 a, 9 \alpha$ & $6 \beta, 15$ & \\
\hline 19 & $4 \beta$ & $1 \alpha, 9 \alpha$ & $6 \beta, 8 \alpha, 15$ & \\
\hline 20 & $4 \beta$ & $9 a$ & $6 \beta, 8 \alpha, 15$ & $1 \alpha(O C i n)$ \\
\hline 21 & $4 \beta$ & $1 \alpha, 9 \alpha$ & $6 \beta, 15$ & $8 \alpha(0 T i g)$ \\
\hline 22 & $4 \beta$ & $9 \alpha$ & $1 \alpha, 6 \beta, 15$ & $8 \alpha(O T i g)$ \\
\hline 23 & $4 \beta$ & $9 \alpha$ & $1 \alpha, 6 \beta, 8 \alpha, 15$ & \\
\hline 24 & $1 \alpha, 4 \beta$ & $9 \alpha$ & $6 \beta, 15$ & $8 \alpha(\mathrm{OMeBut})$ \\
\hline 25 & $4 \beta$ & $8 \alpha, 9 \alpha$ & $1 \alpha, 6 \beta, 15$ & \\
\hline 26 & & $9 \beta$ & $1 \alpha, 2 \alpha, 6 \beta, 8 \alpha, 15$ & \\
\hline 27 & 15 & $9 \beta$ & $1 \alpha, 2 \alpha, 6 \beta, 8 \alpha$ & \\
\hline 28 & $d \beta$ & $1 \alpha, 9 \beta$ & $2 \beta, 3 \beta, 6 \beta$ & \\
\hline 29 & $2 \beta, 4 \beta$ & $1 \alpha, 9 \beta$ & $3 \beta, 6 \beta$ & \\
\hline 30 & $3 \beta, 4 \beta$ & $1 \alpha, 9 \beta$ & $2 \beta, 6 \beta$ & \\
\hline 31 & $3 \beta, 4 \beta$ & $1 \alpha$ & $2 \beta, 6 \beta$ & $9 \beta$ (OCin) \\
\hline 32 & $3 \beta, 4 \beta, 6 \beta$ & $1 \alpha, 9 \beta$ & $2 \beta$ & \\
\hline 33 & $4 \beta$ & $1 \alpha, 9 \beta$ & $2 \beta, 3 \beta, 8 \beta$ & \\
\hline 34 & $4 \beta$ & $9 \alpha$ & $2 \alpha, 6 \beta, 8 \alpha, 15$ & $1 \alpha(O A n g)$ \\
\hline 35 & $4 \beta$ & $1 \alpha, 9 \alpha$ & $2 \alpha, 6 \beta, 8 \alpha, 15$ & \\
\hline 36 & $4 \beta, 6 \beta$ & $1 \alpha, 9 \alpha$ & $2 a, 8 a, 15$ & \\
\hline 37 & $4 \beta$ & $9 \alpha$ & $1 \alpha, 2 \alpha, 6 \beta, 8 \alpha, 15$ & \\
\hline 38 & $4 \beta$ & $1 \alpha, 8 \alpha, 9 \alpha$ & $2 \alpha, 6 \beta, 15$ & \\
\hline 39 & $4 \beta$ & $1 \alpha, 9 \alpha$ & $2 a, 6 \beta, 15$ & $8(C=0)$ \\
\hline 40 & $4 \beta$ & $9 \alpha$ & $1 \alpha, 2 \alpha, 6 \beta, 15$ & $8(C=0)$ \\
\hline 41 & $4 \beta$ & & $1 \alpha, 2 \alpha, 6 \beta, 9 \alpha, 15$ & $\begin{array}{l}8(C=0) \\
3 \beta, 13 \text { (OCass) }\end{array}$ \\
\hline
\end{tabular}

FIG. 1. NATURAL COMPOUNDS ASSAYED. 


\begin{tabular}{lllll} 
& & & \\
& & & \\
\hline
\end{tabular}

FIG. 2. SYNTHETIC COMPOUNDS ASSAYED.

esters, i.e. for compounds with the alatol skeleton the order of activity is 23 (4 OAc; 1 $\mathrm{OBz})>19(3 \mathrm{OAc} ; 2 \mathrm{OBz})>18(2 \mathrm{OAc} ; 3 \mathrm{OBz})$ and $17(3 \mathrm{OAc} ; 1 \mathrm{OBz})>16(2 \mathrm{OAc} ; 2$ $\mathrm{OBz}$ ); for compounds with the $4 \beta$-hydroxyalatol skeleton 37 (5OAc; $1 \mathrm{OBz})>35$ (4 $\mathrm{OAc} ; 2 \mathrm{OBz})>38(3 \mathrm{OAc} ; 3 \mathrm{OBz}$ ). In general, products with aromatic esters at $\mathrm{C}-1$ and C-9, whatever their stereochemistry were only weakly active, i.e. 11-12, 18 and 28. The introduction of ester groups at $\mathrm{C}-2$ had no effect on activity, i.e. in the pairs 18 vs. 38,19 vs. 35 and 23 vs. 37 (Fig. 1 ).

The three insecticidal sesquiterpenes, 39, 40 and orthosphenine (41) showed an $L_{100}$ of $0.1,1$ and $0.01 \mu \mathrm{g} \mathrm{cm}^{-2}$, respectively. The only structural characteristic common to the compounds was a carbonyl group at C-8.

Comparing the antifeedant activity shown by the sesquiterpenes at the concentration of $10 \mu \mathrm{g} \mathrm{cm}^{-2}$ with the well-known antifeedant agent triphenyl tin acetate (TTA, $F R_{50}=0.37$ 土.09), we found that 18 compounds $(3,5-6,8-10,15,17,21-27,34,36$ -37) proved to be more active than TTA, ten compounds $(2,4,11-12,18,28-29,31-$ $32,38)$ were less active and no significant differences were found for ten compounds $(1,7,13-14,16,19-20,30,33,35)$.

No antifeedant nor insecticidal activity was observed in the racemic and/or optically active tetrahydrofuran products with substituents in C-2 or C-3 and 2-methylbutanoid derivatives 42-55 (Fig. 2). All these compounds have a sesquiterpene skeleton and $X$ ray data (González et al., 1992a) and conformational study by molecular mechanics (Jiménez, 1992) showed that in every instance with only slight variations, the transfused $A$ and $B$ rings formed a decalin system, chair-chair, slightly distorted by the presence of the 1,3-diaxial bond responsible for the tetrahydrofuran $C$ ring, practically perpendicular to the plain formed by carbons C-5, C-7, C-8 and C-10.

These results allow us to conclude that the compact tricyclic system is a prerequisite for antifeedant or insecticidal activity in this type of sesquiterpene. 
TABLE 1. ANTIFEEDANT ACTIVITY OF SESQUITERPENES AGAINST Spodoptera littoralis

\begin{tabular}{|c|c|c|}
\hline Compound & Dose $\left(\mu \mathrm{g} \mathrm{cm}^{-2}\right)$ & $\mathrm{FR}_{50} \pm \mathrm{SEM}^{*}$ \\
\hline 1 & 10 & $0.52 \pm 0.10$ \\
\hline 2 & 10 & $0.68 \pm 0.14$ \\
\hline \multirow[t]{3}{*}{3} & 10 & $0.11 \pm 0.04$ \\
\hline & 1 & $0.12 \pm 0.01$ \\
\hline & 0.1 & $0.40 \pm 0.07$ \\
\hline 4 & 10 & $0.74 \pm 0.06$ \\
\hline \multirow[t]{2}{*}{5} & 10 & $0.12 \pm 0.07$ \\
\hline & 1 & $0.40 \pm 0.02$ \\
\hline \multirow[t]{4}{*}{6} & 10 & $0.04 \pm 0.03$ \\
\hline & 1 & $0.15 \pm 0.15$ \\
\hline & 0.1 & $0.45 \pm 0.28$ \\
\hline & 10 & $0.36 \pm 0.09$ \\
\hline \multirow[t]{2}{*}{8} & 10 & $0.19 \pm 0.06$ \\
\hline & 1 & $0.54 \pm 0.09$ \\
\hline \multirow[t]{2}{*}{9} & 10 & $0.04 \pm 0.02$ \\
\hline & 1 & $0.44 \pm 0.09$ \\
\hline \multirow[t]{2}{*}{10} & 10 & $0.05 \pm 0.01$ \\
\hline & 1 & $0.25 \pm 0.06$ \\
\hline 11 & 10 & $0.61 \pm 0.09$ \\
\hline 12 & 10 & $0.63 \pm 0.11$ \\
\hline 13 & 10 & $0.35 \pm 0.08$ \\
\hline 14 & 10 & $0.41 \pm 0.11$ \\
\hline \multirow[t]{2}{*}{15} & 10 & $0.16 \pm 0.15$ \\
\hline & 1 & $0.65 \pm 0.25$ \\
\hline 16 & 10 & $0.45 \pm 0.12$ \\
\hline \multirow[t]{4}{*}{17} & 10 & 0 \\
\hline & 1 & 0 \\
\hline & 0.1 & $0.23 \pm 0.07$ \\
\hline & 0.01 & $0.69 \pm 0.08$ \\
\hline 18 & 10 & $0.76 \pm 0.08$ \\
\hline \multirow[t]{2}{*}{19} & 10 & $0.24 \pm 0.11$ \\
\hline & 1 & $0.58 \pm 0.06$ \\
\hline 20 & 10 & $0.27 \pm 0.16$ \\
\hline \multirow[t]{2}{*}{21} & 10 & $0.12 \pm 0.03$ \\
\hline & 1 & $0.29 \pm 0.07$ \\
\hline \multirow[t]{3}{*}{22} & 10 & $0.06 \pm 0.03$ \\
\hline & 1 & $0.20 \pm 0.06$ \\
\hline & 0.1 & $0.43 \pm 0.06$ \\
\hline \multirow[t]{3}{*}{23} & 10 & $0.03 \pm 0.01$ \\
\hline & 1 & $0.20 \pm 0.06$ \\
\hline & 0.1 & $0.64 \pm 0.07$ \\
\hline \multirow[t]{3}{*}{24} & 10 & $0.08 \pm 0.03$ \\
\hline & 1 & $0.23 \pm 0.02$ \\
\hline & 0.1 & $0.57 \pm 0.09$ \\
\hline \multirow[t]{2}{*}{25} & 10 & $0.15 \pm 0.02$ \\
\hline & 1 & $0.40 \pm 0.09$ \\
\hline \multirow[t]{2}{*}{26} & 10 & $0.07 \pm 0.06$ \\
\hline & 1 & $0.53 \pm 0.32$ \\
\hline \multirow[t]{2}{*}{27} & 10 & $0.13 \pm 0.05$ \\
\hline & 1 & $0.71 \pm 0.09$ \\
\hline 28 & 10 & $0.65 \pm 0.15$ \\
\hline 29 & 10 & $0.56 \pm 0.11$ \\
\hline \multirow[t]{2}{*}{30} & 10 & $0.24 \pm 0.19$ \\
\hline & 1 & $0.44 \pm 0.12$ \\
\hline 31 & 10 & $0.76 \pm 0.16$ \\
\hline 32 & 10 & $0.60 \pm 0.07$ \\
\hline 33 & 10 & $0.38 \pm 0.05$ \\
\hline
\end{tabular}




\begin{tabular}{lcc}
\hline Compound & Dose $\left(\mu \mathrm{g} \mathrm{cm}^{-2}\right)$ & FR $_{\mathbf{5 0}} \pm$ SEM* \\
\hline $\mathbf{3 4}$ & 10 & $0.10 \pm 0.03$ \\
& 1 & $0.18 \pm 0.02$ \\
$\mathbf{3 5}$ & 0.1 & $1.10 \pm 0.02$ \\
$\mathbf{3 6}$ & 10 & $0.35 \pm 0.19$ \\
& 10 & $0.10 \pm 0.06$ \\
$\mathbf{3 7}$ & 1 & $0.19 \pm 0.08$ \\
& 0.1 & $1.02 \pm 0.07$ \\
& 10 & $0.05 \pm 0.02$ \\
$\mathbf{3 8}$ & 1 & $0.24 \pm 0.03$ \\
TTAt & 0.1 & $0.40 \pm 0.08$ \\
\hline
\end{tabular}

*Standard Error Mean.

tTriphenyl tin acetate.

Sesquiterpenes with the basic dihydro- $\beta$-agarofuan skeleton represent a new family of active compounds with potential for use as crop protection agents in agriculture.

Acknowledgements-This work has been subsidized by $\mathrm{ClCYT}$ Projects SAF 92-1028-CO2-01 and BI 088-0230 and the Colegio Libre de Eméritos (Madrid).

\section{References}

Bellés, X. and Piulachs, M. D. (1983) Actividad Fagorepelente del Acetato de Trifenilestaño sobre Larvas de Spodoptera littoralis (Boisduval) (Lepidoptera noctuidae). Act. / Congreso Ibérico Entomol., Vol. 1, pp. 101-105. León, España.

Bellés, X., Camps, F., Colt, J and Piulachs, M. D. (1985) Insect antifeedant activity of clerodane diterpenoids on larvae of Spodoptera littoralis (Boisd.) (Lepodoptera). J. Chem. Ecol. 11, 1439-1445.

Brüning, R. and Wagner, H. (1978) Ubersicht Über Die Celastraceen Inhalstsstoffe: Chemie, Chemotaxonomie, Biosynthese, Pharmakologie. Phytochemistry 17, 1821-1858.

TABLE 2. BASTC POLYHYDROXY SKELETONS OF THE SESOUITERPENES ASSAYED

\begin{tabular}{|c|c|c|}
\hline Compound & Basic skeleton & No. oxygens \\
\hline 1 & Boariol & 3 \\
\hline 2 & $4 \beta$-Hydroxycelorbicol & 5 \\
\hline 3 & 2,3-Dideoxymaytol & 6 \\
\hline 4-6 & $2,3,13,15-$ Tetradeoxyisoeuonyminol & 6 \\
\hline 7-8 & 3,4-Dideoxymaytol & 6 \\
\hline $9-10$ & 15-Hydroxycelapanol & 6 \\
\hline 11 & 6-Deoxymagellanol & 6 \\
\hline 12 & 4.Deoxymagellanol & 6 \\
\hline 13 & 3-Deoxymagellanol & 6 \\
\hline 14 & 3,4,13-Trideoxyevoninol & 7 \\
\hline 15 & Alatol & 7 \\
\hline 16-25 & Isoalatol & 7 \\
\hline $26-27$ & 9-Epialatol & 7 \\
\hline $28-32$ & Magellanol & 7 \\
\hline 33 & Isomagellanol & 7 \\
\hline $34-38$ & $4 \beta-$ Hydroxyalatol & 8 \\
\hline $39-40$ & 3,13-Dideoxyevoninol & 8 \\
\hline 41 & Evoninol & 10 \\
\hline
\end{tabular}


Chiu, S. F. (1989) Recent advances in research on botanical insecticides in China. A.C.S. Symp. Ser. 387, 6977.

Geuskens, R. B. M., Lutejin, J. M. and Schoonhoven, L. M. (1983) Antifeedant activity of some ajugarin derivatives in three lepidopterous species. Experientia 39, $403-404$.

González, A. G., González, C. M., Bazzocchi, I. L., Ravelo, A. G., Luis, J. G. and Dominguez, X. A. (1987) Sesquiterpene alkaloids from the Celastraceae. Phytochemistry 26, 2133-2135.

González, A. G., González, C. M., Ravelo, A. G., Fraga, B. M. and Domínguez, X. A. (1988) Sesquiterpenes from Orthosphenia mexicana. Phytochemistry 27, 473-477.

González, A. G., Jiménez, I. A., Ravelo, A. G., Luis, J. G. and Bazzocchi, I. L. (1989a) Agarofurane sesquiterpene esters from Maytenus canariensis. Phytochemistry 28, 173-175.

González, A. G., San Andrés, L., Ravelo, A. G., Luis, J. G., Jiménez, I. A. and Domínguez, X. A. (1989b) Minor constituents from Orthosphenia mexicana. J. Nat. Prod. 52, 1338-1341.

González, A. G., Jiménez, I. A., Ravelo, A. G. and Bazzocchi, I. L. (1990) B-Agarofuran sesquiterpenes from Maytenus canariensis. Phytochemistry 29, 2577-2579.

González, A. G., Jiménez, I. A., Ravelo, A. G., Bellés, X. and Piulachs, M. D. (1992a) Antifeedant activity of dihydro- $\beta$-agarofuran sesquiterpenes from Celastraceae against Spodoptera littoralis. Biochem. Syst. Ecol. 20 , 311-315.

González, A. G., Muñoz, O., Ravelo, A. G., Crespo, A., Bazzocchi, I. L., Jiménez, I. A., Solans, X., Ruiz-Pérez, C. and Rodríguez-Romero, V. (1992b) A new sesquiterpene from Maytenus boaria (Celastraceae) crystal structure and absolute configuration. Tetrahedron Lett. 33, 1921-1924.

González, A. G., Jiménez, I. A., Ravelo, A. G., Gavin, S. J. and Bazzocchi, I. L. (1993a) New sesquiterpenes with antifeedant activity from Maytenus canariensis (Celastraceae). Tetrahedron 49, 697-702.

González, A. G., Jiménez, I. A., Ravelo, A. G. and Bazzocchi, I. L. (1993b) Minor sesquiterpenes from Maytenus canariensis with insecticidal and antifeedant activity. Tetrahedron 49, 6637-6644.

González, A. G., Jiménez, I. A., Nuñez, M. P., Ravelo, A. G., Bazzocchi, I. L., Muñoz, O. M. and Aguilar, M. A. (1994a) New sesquiterpenes from Maytenus species (Celastraceae). Taxonomic and chemotaxonomic considerations concerning Chitean Maytenus. J. Chem. Ecol. 20, 823-830.

González, A. G., Jiménez, I. A., Ravelo, A. G. and Bazzocchi, I. L. (1994b) New angelic and tiglic dihydro- $\beta-$ agarofuran ester sesquiterpenoids from Maytenus canariensis (Celastraceae). Natural Product Lett. 5, 33-40.

Jiménez, I. A. (1992) Metabolitos Secudarios Bioactivos de Celastraceas (Maytenus boaria, M. canariensis y Orthosphenia mexicana). Doctoral Thesis, Universidad de La Laguna.

Kai, L. J., Wen, H. X., Jian, J. Z., Yong, J. and Qin, W. H. (1991) Two sesquiterpene alkaloids from Celastrus angulatus. Phytochemistry 30, 3437-3440.

Kato, N., Takahashi, M., Shibayama, M. and Munakata, K. (1972) Antifeeding active substances for insects in Clerodendrom tricotomum Thumb. Agric. Biol. Chem. 36, 2579-2582.

Kojima, Y. and Kato, N. (1981) Stereocontrolled synthesis of clerodin homolog: a synthetic approach to structure-activity relationships. Tetrahedron 37, 2527-2538.

Kubo, I., Lee, Y. W., Pettei, M. J., Piliewicz, F. and Nakanishii, K. (1976) Potent army worm antifeedants from the East African Warbugia plant. J. Chem. Soc. Chem. Commun. 1013-1014.

Ley, S. V. (1990) Synthesis and modification of azadiractin and related antifeedants. Recent advances in the chemistry of insects control II. Royal Society of Chemistry. Special Publ. 79, 90-98.

Maotian, W., Hailin, Q., Man, K. and Janzi, L. (1991) Insecticidal sesquiterpene polyol ester from Celastrus angulatus. Phytochemistry 30, 3931-3933.

Poitout, S. and Bues, R. (1974) Elevange de Chenilles de Vingt-huit Espèces de Lépidoptères (Noctuidae) et de Deux Espèces de Arctiidae sur Milieu Aritificiel Simple. Particularités de l'Elevage Selon les Espèces. Ann. Zoo. Ecol. Anim. 6 (3), 431-441.

Swingle, W. T., Haller, H. L., Siegler, E. H. and Swingle, M. C. (1941) A Chinese insecticidal plant, Tripterigium wiffordii, introduced into the United States. Science 93, 60-61.

Wakabayashi, N., Wu, W. J., Waters, R. M., Redfern, R. E., Mills, G. D., De Milo, A., Lusby, W. R. and Andrzejewski, D. (1988) Celangulin: a nonalkaloidal insect antifeedant from Chinese bittersweet, Celastrus angulatus. J. Nat. Prod. 51, 537-542.

Wu, W. J., Tu, Y. Q., Liu, H. X. and Xhu, J. B. (1992) Celangulins II, III and IV: new insecticidal sesquiterpenoids from Celastrus angulatus. J. Nat. Prod. 55, 1294. 